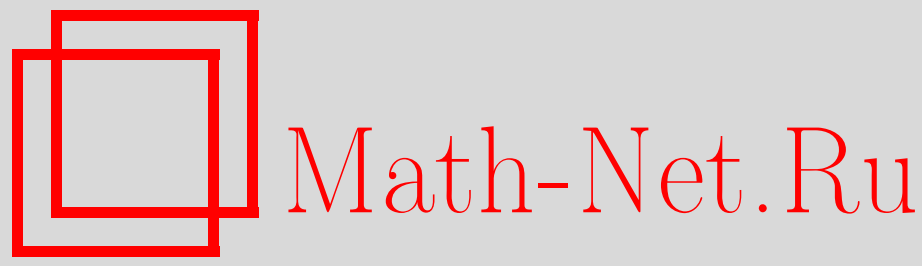

И. Я. Арефьева, И. В. Волович, Об изотропном энергетическом условии и космологии, ТМФ, 2008, том 155 , номер 1, 3-12

DOI: https://doi.org/10.4213/tmf6188

Использование Общероссийского математического портала Math-Net.Ru подразумевает, что вы прочитали и согласны с пользовательским соглашением http://www.mathnet.ru/rus/agreement

Параметры загрузки:

IP: 3.89.185.249

26 апреля 2023 г., 18:08:34

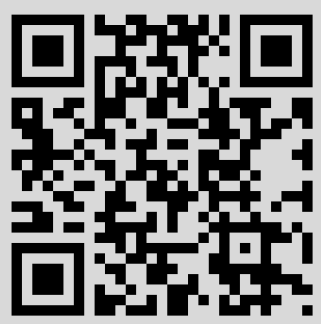




\title{
ОБ ИЗОТРОПНОМ ЭНЕРГЕТИЧЕСКОМ УСЛОВИИ И КОСМОЛОГИИ
}

\begin{abstract}
Полевые теории, которые нарушают изотропное энергетическое условие, представляют интерес как для решения проблемы космологической сингулярности, так и для моделей космологической темной энергии с параметром уравнения состояния $w<-1$. Рассмотрены две недавно предложенные модели, которые нарушают это условие. Модель фантомного конденсата требует высших производных, что приводит к появлению массивного фантома и не ограниченной снизу энергии. Дается оценка скорости распада частиц и обсуждаются возможные ограничения на массы, необходимые для того, чтобы обеспечить стабильность частиц в модели фантомного конденсата. Нелокальная струнная модель, которая появляется в кубической полевой струнной теории и проявляет фантомное поведение, также приводит к не ограниченной снизу энергии. В этом случае спектр энергии непрерывен и частицеподобные возбуждения отсутствуют. Эта модель допускает естественное ультрафиолетовое пополнение, так как она выводится из теории суперструны.
\end{abstract}

Ключевые слова: космология, струны, D-браны.

\section{1. ВВЕДЕНИЕ}

Существуют общие ограничения на тензор энергии-импульса физических систем так называемые энергетические условия. Они играют важную роль в общей теории относительности, в особенности при рассмотрении черных дыр и космологических особенностей (см. работы [1]-[7] и приведенные там ссылки).

В слабой форме энергетическое условие утверждает, что $T_{\mu \nu} n^{\mu} n^{\nu} \geqslant 0$ для всякого нуль-вектора $n^{\mu}$; далее мы будем использовать английское сокращение NEC null energy condition. Это условие непосредственно связано с ограничением на параметр $w=p / \rho$ уравнения состояния, где $p$ - давление, а $\rho-$ энергия. Тензор энергии-импульса для пространственно-однородной изотропной материи имеет диагональный вид $T_{\mu \nu}=\operatorname{diag}(\rho, p, p, p)$. Условие $w<-1$ для материи с положительной плотностью энергии $\rho$ подразумевает нарушение NEC [8], [9]. Поскольку экспериментальные данные не исключают возможности того, что $w<-1$ для темной

${ }^{*}$ Математический институт им. В. А. Стеклова РАН, Москва, Россия.

E-mail: arefeva@mi.ras.ru, volovich@mi.ras.ru 
энергии, на долю которой по современным данным приходится около $70 \%$ всего вещества, исследование таких моделей привлекает большое внимание ${ }^{1)}$. В моделях, в которых имеет место так называемый “отскок” от космологической сингулярности, NEC также нарушается [2], [11].

Существуют общие теоремы, которые утверждают, что если в модели гравитации, взаимодействующей со скалярными полями, нарушено NEC, то такая модель нестабильна [3]-[5], [7], [12]. Однако недавно были сделаны попытки построить модели, к которым эти общие теоремы не применимы. Это модель фантомного конденсата [13]-[16] и нелокальная струнная модель [17]-[20], которые включают в себя высшие производные. В нашей работе мы анализируем эти две модели, используя метод Остроградского и представление Вейерштрасса.

Мы показываем, что модель фантомного конденсата в силу присутствия слагаемых с высшими производными, вообще говоря, приводит к массивному фантому и не ограниченной снизу энергии, и в результате этого нарушается стабильность вещества. Мы даем оценку скорости распада частиц и обсуждаем возможные ограничения на значения масс, обеспечивающие стабильность материи в модели фантомного конденсата.

Нелокальная струнная модель, которая проявляет фантомное поведение на определенных участках эволюции, также приводит к не ограниченной снизу энергии, но в этом случае спектр энергии непрерывен и частицеподобные возбуждения отсутствуют. Эта модель допускает естественное ультрафиолетовое пополнение, так как она связана с теорией суперструны.

Более детально, рассматриваемая нелокальная струнная модель основана на струнно-полевой теории фермионной NSR-струны с GSO-сектором [21]. В этой модели скалярное поле - это тахион открытой струны [22], который описывает согласно гипотезе Сена [23] динамический переход неэкстремальной D-браны к устойчивому вакууму (см. обзоры [24]). Эта устойчивая модель должна описываться вакуумной струнной полевой теорией. Изучаемая нами нелокальная струнная модель представляет собой приближение к вакуумной струнной полевой теории, которая является устойчивой и не имеет никаких частицеподобных возбуждений, соответствующих открытой струне.

Векторно-скалярная модель, нарушающая NEC, с возбуждениями, устойчивыми в некоторой области, была предложена в [25]. Существует скалярно-тензорная модель темной энергии, допускающая фантомное поведение в области малых красных смещений [26]. Модели темной энергии, так или иначе связанные со струнами и бранами, интенсивно исследовались в последние годы (см., например, [27]-[29] и приведенные там ссылки).

Статья организована следующим образом. В разделе 2 мы обсуждаем модель фантомного конденсата. Мы показываем, что эта модель фактически включает в себя два нерелятивистских скалярных поля. Одно поле - это массивный фантом, в то время как другое поле имеет положительную энергию. Мы оцениваем время распада обычной частицы в конденсате и получаем довольно сильное ограничение на массу частицы, наложив требование, чтобы время жизни частицы было порядка

1)В работе [10] была предложена стратегия прямых экспериментов по проверке неравенства $w<-1$. 
времени жизни Вселенной. В разделе 3 мы рассматриваем нелокальную струнную модель. Чтобы найти спектр модели, мы используем представление свободной части действия в виде произведения Вейерштрасса и метод Остроградского. Мы получаем, что для этой модели энергия является не ограниченной снизу, но в этом случае отсутствуют возбуждения типа частиц.

\section{2. МОДЕЛЬ ФАНТОМНОГО КОНДЕНСАТА}

2.1. Описание модели. В модели фантомного конденсата в плоском четырехмерном пространстве Минковского с сигнатурой $(-,+,+,+)$ используется лагранжиан [13]-[16]

$$
\mathcal{L}=M^{4} P(X)+M^{2} S_{1}(X)(\square \phi)^{2}+M^{2} S_{2}(X) \partial^{\mu} \partial^{\nu} \phi \partial_{\mu} \partial_{\nu} \phi
$$

Здесь $\phi-$ скалярное поле,

$$
\begin{aligned}
X & =-\partial_{\mu} \phi \partial^{\mu} \phi=\dot{\phi}^{2}-(\nabla \phi)^{2}, \\
\square \phi & =-\partial_{0}^{2} \phi+\nabla^{2} \phi
\end{aligned}
$$

и $M$ - произвольный массовый параметр. Фантом может проявляться вследствие наличия слагаемого $P(X)$. В лагранжиан также входят слагаемые с высшими производными $S_{1}$ и $S_{2}$, которые, как мы увидим ниже, также приводят к фантомам.

Если

$$
P(X)=\frac{1}{8}(X-1)^{2}=-\frac{1}{4} \dot{\phi}^{2}+\cdots,
$$

то кинетический член имеет "неправильный" знак, и появляются фантомы. Это соответствует "неправильному" вакуумному решению полевых уравнений

$$
\phi_{0}=0 \text {. }
$$

В работе [13] было предложено по аналогии с механизмом Хиггса рассматривать теорию в окрестности нетривиального вакуума; при этом конфигурации с $P^{\prime}(X)=0$ рассматривались в качестве основного состояния. Имеется решение полевых уравнений $P^{\prime}(X)=0$ вида

$$
\phi_{0}=t \text {. }
$$

При рассмотрении малых флуктуаций $\pi(t, x)$ в окрестности этого решения,

$$
\phi=t+\pi(t, x)
$$

лагранжиан (4) для квадратичного приближения имеет “правильный” знак перед кинетическим членом:

$$
\mathcal{L}=\frac{1}{2} M^{4} \dot{\pi}^{2}
$$

Заметим, что получившийся лагранжиан не содержит квадратичного члена с пространственной производной $(\nabla \pi)^{2}$. Члены старшего порядка $S_{1}$ и $S_{2}$ в $(1)$ добавлены к $P$, чтобы обеспечить наличие слагаемого с пространственной производной. Следующий квадратичный лагранжиан рассматривался в [13]-[16]:

$$
\mathcal{L}=\frac{1}{2} M^{4} \dot{\pi}^{2}-\frac{1}{2} \gamma^{2}\left(\nabla^{2} \pi\right)^{2}
$$


где $\gamma^{2}$ - постоянная,

$$
\gamma^{2}=-2 M^{2}\left(S_{1}(1)+S_{2}(1)\right)
$$

Отметим, однако, что исходный лагранжиан (1) приводит не к формуле (9), а к следующему квадратичному лагранжиану:

$$
\mathcal{L}=\frac{1}{2} M^{4} \dot{\pi}^{2}-\frac{1}{2} \gamma^{2}(\square \pi)^{2}
$$

где $\square \pi=-\ddot{\pi}+\nabla^{2} \pi$. Этот лагранжиан включает члены с высшими производными, что порождает духи для всех импульсов. В действительности он описывает не одно, а два скалярных поля. Чтобы показать это, рассмотрим теории с высшими производными.

\section{2. Метод Остроградского для уравнений с высшими производными.} Рассмотрим лагранжиан

$$
\mathcal{L}=\varphi \square(\alpha+\beta \square) \varphi
$$

где $\alpha$ и $\beta$ - вещественные параметры. Используя метод Остроградского [30], [31], введем поля

$$
\psi=(\alpha+\beta \square) \varphi, \quad \phi=\square \varphi .
$$

С точностью до слагаемых типа дивергенции лагранжиан (12) может быть записан в виде

$$
\mathcal{L}=\frac{1}{\alpha} \psi \square \psi-\frac{\beta}{\alpha} \phi(\alpha+\beta \square) \phi .
$$

Мы видим, что независимо от знаков $\alpha$ и $\beta$ одно из полей $\phi$ и $\psi$ является фантомом. Например, если $\alpha>0$ и $\beta<0$, то мы имеем бесмассовую частицу $\psi$ и массивный фантом $\phi$.

Случай лагранжиана

$$
\mathcal{L}=\varphi \square^{2} \varphi
$$

более сложен для анализа. Предел $\alpha \rightarrow 0$ сингулярен, чтобы с ним работать, надо сначала выполнить каноническое преобразование [32], [33].

2.3. Распад частиц. Фантомы имеют отрицательную энергию. Поэтому обычные частицы могут распасться на более тяжелые частицы и фантомы. Это обсуждается в [3], [4].

Мы хотим оценить время жизни обычной частицы относительно таких распадов. Прямое взаимодействие, описывающее этот распад, отсутствует. В нашей модели имеется взаимодействие фантома с гравитацией и взаимодействие гравитации с обычными частицами. Простая диаграмма, описывающая распад обычной частицы $\psi$ на обычные частицы $\psi$ и $\chi$ и два фантома $\phi$, представлена на рисунке $(h-$ гравитон).

Мы предполагаем, что взаимодействия фантом-гравитация и обычные частицы-гравитация происходят только из массовых членов в действии

$$
\begin{array}{r}
S=M_{p}^{2} \int d^{4} x \sqrt{-g} R+\frac{1}{2} \int d^{4} x \sqrt{-g}\left[(\partial \phi)^{2}+M^{2} \phi^{2}-\right. \\
\left.-(\partial \psi)^{2}-m_{\psi}^{2} \psi^{2}-(\partial \psi)^{2}-M_{\chi}^{2} \chi^{2}+\lambda_{\psi^{2}} \psi^{2} \chi\right]
\end{array}
$$




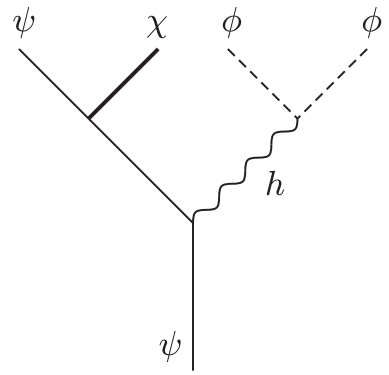

Распад $\psi$ частицы на два фантома $\phi$ и две обычные частицы $\psi$ и $\chi$.

Здесь $\phi$ - фантом, а $\psi$ и $\chi$ - обычные частицы. Предполагается, что есть кубическое взаимодействие обычных частиц между собой, но с фантомом они взаимодействуют только через гравитацию. Мы также предполагаем, что масса $M_{\chi}$ частицы $\chi$ имеет порядок массы фантома $M$, а масса $m_{\psi}$ частицы $\psi$ намного меньше. Из этого действия мы получаем взаимодействие гравитации со скалярными полями:

$$
\begin{aligned}
\sqrt{-g} \frac{M^{2}}{2} \phi^{2} & \Rightarrow \frac{M^{2}}{M_{p}} \phi^{2} h, \\
\sqrt{-g} \frac{m_{\psi}^{2}}{2} \psi^{2} & \Rightarrow \frac{m_{\psi}^{2}}{M_{p}} \psi^{2} h,
\end{aligned}
$$

где скалярное поле $h$ символически представляет гравитон. Следовательно, константы взаимодействия гравитации с полями $\phi$ и $\psi$ даются выражениями

$$
\lambda_{h \phi^{2}}=\frac{M^{2}}{M_{p}}, \quad \lambda_{h \psi^{2}}=\frac{m_{\psi}^{2}}{M_{p}} .
$$

Полуширина распада частицы на фантомы через канал (см. рисунок),

$$
\psi \rightarrow \psi+\chi+\phi_{1}+\phi_{2},
$$

есть

$$
\begin{aligned}
\Gamma= & \frac{1}{m_{\psi}} \int^{\Lambda} \frac{d^{3} p_{\phi_{1}}}{(2 \pi)^{3} 2 E_{\phi_{1}}} \frac{d^{3} p_{\phi_{2}}}{(2 \pi)^{3} 2 E_{\phi_{2}}} \frac{d^{3} p_{\psi}}{(2 \pi)^{3} 2 E_{\psi}} \frac{d^{3} p_{\chi}}{(2 \pi)^{3} 2 E_{\chi}}|\mathcal{M}|^{2} \times \\
& \times(2 \pi)^{4} \delta^{(4)}\left(p_{\psi_{\text {in }}}-p_{\phi_{1}}-p_{\phi_{2}}-p_{\psi}-p_{\chi}\right),
\end{aligned}
$$

где матричный элемент имеет вид

$$
\mathcal{M}=\lambda_{h \phi^{2}} \lambda_{h \psi^{2}} \lambda_{\chi \psi^{2}} \frac{1}{p^{2}} \frac{1}{q^{2}+m_{\psi}^{2}} .
$$

В (21) $\Lambda$ - обрезание по импульсам, $p$ - импульсы внутренней линии гравитона и $q$ - импульсы внутренней линии частицы $\psi$. Мы предполагаем, что размерная постоянная взаимодействия $\lambda_{\chi \psi^{2}}$ имеет порядок $m_{\psi}, \lambda_{\chi \psi^{2}} \sim m_{\psi}$. Используя (17) и (18), получаем

$$
\mathcal{M}=\frac{m_{\psi}^{3} M^{2}}{M_{p}^{2}} \frac{1}{p^{2}} \frac{1}{q^{2}+m_{\psi}^{2}} .
$$


Интеграл в (21) является сходящимся даже без обрезания. Чтобы его оценить, мы можем взять $M \sim M_{p} \sim \Lambda$, при этом мы получаем оценку для полуширины распада $Г$ :

$$
\Gamma \sim \frac{m_{\psi}^{5}}{M_{p}^{5}} M_{p}
$$

Отсюда можно оценить время жизни частицы $\tau_{\psi}=1 / \Gamma$. Модель считается феноменологически приемлемой, если время жизни больше, чем время жизни Вселенной $H_{0}^{-1}$, т.е.

$$
\Gamma \sim \frac{m_{\psi}^{5}}{M_{p}^{5}} M_{p}<H_{0} \sim 10^{-60} M_{p} .
$$

С учетом того, что $M_{p} \sim 10^{19}$ ГэВ, мы получаем

$$
m_{\psi}<10^{7} \Gamma \ni \mathrm{B}, \quad \Gamma \sim \frac{m_{\psi}^{3}}{M_{p}^{3}} M_{p},
$$

и это дает неприемлемый результат

$$
m_{\psi}<0.1 \Gamma \ni \mathrm{B}
$$

\section{3. НЕЛОКАЛЬНАЯ СТРУННАЯ МОДЕЛЬ}

\section{1. Пример полевого уравнения с экспоненциальным кинетическим} членом. Рассмотрим следующий лагранжиан:

$$
\mathcal{L}=\phi\left(e^{\square}-1\right) \phi
$$

Полевые уравнения для этого лагранжиана нелокальны, их можно записать как интегральные уравнения. По поводу строгого математического подхода к таким уравнениям см. недавние работы [34].

Используя произведение Вейерштрасса

$$
e^{z}-1=e^{z / 2} z \prod_{j=1}^{\infty}\left(1+\frac{z^{2}}{\omega_{j}^{2}}\right),
$$

где $z$ - комплексная переменная, $\omega_{j}^{2}=4 \pi^{2} j^{2}$, мы записываем

$$
e^{\square}-1=e^{\square / 2} \square \prod_{j=1}^{\infty}\left(1+\frac{\square^{2}}{\omega_{j}^{2}}\right) .
$$

Согласно [32] лагранжиан (28) имеет тот же спектр, что и лагранжиан

$$
\mathcal{L}=\psi_{0} \square \psi_{0}+\sum_{j=1}^{\infty} \epsilon_{j} \psi_{j}\left(\square^{2}+\omega_{j}^{2}\right) \psi_{j}
$$

Здесь

$$
\epsilon_{j}=\frac{1}{\omega_{j}^{4}} F^{\prime}\left(-\omega_{j}^{2}\right)
$$


где

$$
F\left(z^{2}\right)=\left(e^{z}-1\right) \frac{e^{-z / 2}}{z}=2 \frac{\operatorname{sh}(z / 2)}{z} .
$$

Более общая функция Лагранжа

$$
\mathcal{L}=\phi\left(e^{\alpha \square}-\lambda\right) \phi
$$

появляется в теории р-адической струны (см. [35]-[37]). Здесь $\alpha$ и $\lambda$ - константы, $\lambda>0$. Спектр теории может быть получен из произведения Вейерштрасса

$$
e^{\alpha \square}-\lambda=\lambda^{1 / 2} e^{\alpha \square / 2}(\alpha \square-\ln \lambda) \prod_{j=1}^{\infty}\left(1+\frac{(\alpha \square-\ln \lambda)^{2}}{\omega_{j}^{2}}\right) .
$$

3.2. Нелокальное тахионное поле. Эффективный лагранжиан для поля тахиона в GSO-секторе фермионной NSR-струны, динамика которой описывается в соответствии с кубической полевой теорией [21], имеет следующий вид [22]:

$$
\mathcal{L}=\frac{1}{2} \phi e^{-\square / 4}\left(\square+m^{2}\right) \phi-\frac{\lambda}{4} \phi^{4}
$$

Тривиальное решение полевых уравнений, соответствующее нестабильному вакууму, имеет вид $\phi_{0}=0$, и в окрестности этого вакуума в линейном приближении получаем тахионное уравнение

$$
\left(\square+m^{2}\right) \phi=0
$$

Также существует другое вакуумное решение

$$
\phi_{0}=\frac{m}{\sqrt{\lambda}}
$$

В этом случае в линейном приближении получается следующий лагранжиан для малых флуктуаций:

$$
\mathcal{L}=\frac{m^{2}}{2} \phi F(-\square) \phi
$$

где

$$
F(z)=\left(-\xi^{2} z+1\right) e^{z / 4}-3
$$

и $\xi^{2}=1 / m^{2}$.

Характеристическое уравнение

$$
F(z)=0
$$

имеет бесконечное число комплексно-сопряженных корней $z=\kappa_{j}^{2}$ (см. работу [20], где рассмотрены эти корни в случае действия, заданного формулами (39), (40), и работу [38] для случая произвольных констант). Корни $\kappa_{j}^{2}$ определяются с помощью функции Ламберта $W(z)$, удовлетворяющей уравнению $W(z) e^{W(z)}=z$, а именно $\kappa_{j}^{2}=1 / \xi^{2}+4 W_{j}\left(-3 e^{-1 / 4 \xi^{2}} / 4 \xi^{2}\right)$, где $W_{j}-j$-я ветвь функции Ламберта [20], [38]. 
Представляем (40) как произведение Вейерштрасса:

$$
F(z)=-2 e^{\left(4 \xi^{2}-1\right) z / 8} \prod_{j}\left(1-\frac{z}{\kappa_{j}^{2}}\right)\left(1-\frac{z}{\left(\kappa_{j}^{*}\right)^{2}}\right) \exp \left[z\left(\frac{1}{\kappa_{j}^{2}}+\frac{1}{\left(\kappa_{j}^{*}\right)^{2}}\right)\right] .
$$

Теперь мы можем записать (с точностью до полных производных) соответствующую квадратичную функцию Лагранжа как

$$
\mathcal{L}=\sum_{j} \epsilon_{j} \psi_{j}\left(\square-\kappa_{j}^{2}\right)\left(\square-\left(\kappa_{j}^{*}\right)^{2}\right) \psi_{j} .
$$

Системы с различными комплексными частотами были изучены в [32]. Рассмотрим следующее уравнение:

$$
\left(\square-\kappa^{2}\right)\left(\square-\left(\kappa^{*}\right)^{2}\right) \phi=0
$$

где $\kappa=\nu+i \alpha$. Соответствующий гамильтониан является линейной суперпозицией пары комплексно-сопряженных осцилляторов:

$$
H=\frac{1}{2} \sum_{k}\left[P_{1}^{2}(k)+\omega(k)^{2} Q_{1}^{2}(k)+P_{2}^{2}(k)+\left(\omega(k)^{*}\right)^{2} Q_{2}^{2}(k)\right],
$$

где $P_{1}^{*}=P_{2}, Q_{1}^{*}=Q_{2}$ и $\omega(k)^{2}=\kappa^{2}+k^{2}$. Спектр гамильтониана имеет вид

$$
n \nu(k)+\rho \alpha(k)
$$

где мы положили $\omega(k)=\nu(k)+i \alpha(k)$. Здесь $n=0, \pm 1, \ldots$, в то время как $\rho$ непрерывна и изменяется от $-\infty$ до $+\infty$. Энергетический спектр не ограничен снизу и непрерывен. Следовательно, в этой модели частицеподобные возбуждения отсутствуют.

\section{4. ЗАКЛЮЧЕНИЕ}

Мы рассмотрели две недавно предложенные модели, которые нарушают NEC. В обеих моделях имеются высшие производные, и мы использовали представление Вейерштрасса и метод Остроградского, чтобы изучить эти модели. Применение данных методов к линейным теориям показывает, что энергии не ограничены снизу.

Отметим, что проблема с не ограниченной снизу энергией, которая рассматривается в этой статье, математически подобна проблеме исследования не ограниченных снизу собственных значений для гиперболического оператора Клейна-Гордона на лоренцевых многообразиях [39].

Можно ожидать, что рассмотрение нелинейных уравнений могло бы существенно изменить ситуацию. В частности, в нелинейных теориях могли бы существовать острова стабильности [3], [40]-[42].

В модели фантомного конденсата с высшими производными было бы интересно найти ультрафиолетовое пополнение теории и исследовать стабильность соответствующей теории. 
Нелокальная струнная модель, описывающая распад D-бран, также приводит к не ограниченной снизу энергии, однако в этом случае спектр энергии непрерывен и отсутствуют частицеподобные возбуждения. Эта модель допускает ультрафиолетовое пополнение, так как она получается из теории суперструны, и было бы очень интересно найти прямой механизм компенсации не ограниченного снизу непрерывного спектра.

Благодарности. Работа частично поддержана INTAS (грант № 03-51-6346). И. Я. Арефьева частично поддержана РФФИ (грант № 05-01-00758) и Программой поддержки ведущих научных школ (грант № НШ-2052.2003.1). И. В. Волович частично поддержан РФФИ (грант № 05-01-00884) и Программой поддержки ведущих научных школ (грант № НШ-1542.2003.1).

\section{Список литературы}

[1] S. W. Hawking, G. F. R. Ellis, The Large Scale Structure of Space-Time, Cambridge University Press, London, 1973.

[2] M. Gasperini, G. Veneziano, Phys. Rep., 373:1-2 (2003), 1-212; arXiv: hep-th/0207130.

[3] S. M. Carroll, M. Hoffman, M. Trodden, Phys. Rev. D, 68:2 (2003), 3509; arXiv: astro-ph/0301273.

[4] J. M. Cline, S. Jeon, G. D. Moore, Phys. Rev. D, 70:4 (2004), 3543; arXiv: hep-ph/0311312.

[5] S. D. H. Hsu, A. Jenkins, M. B. Wise, Phys. Lett. B, 597:3-4 (2004), 270-274; arXiv: astro-ph/0406043.

[6] B. McInnes, Nucl. Phys. B, 718:1-2 (2005), 55-82; arXiv: hep-th/0502209.

[7] R. V. Buniy, S. D. H. Hsu, B. M. Murray, Phys. Rev. D, 74:6 (2006), 3518; arXiv: hep-th/0606091.

[8] E. J. Copeland, M. Sami, Sh. Tsujikawa, Internat. J. Modern Phys. D, 15:11 (2006), 1753-1935; arXiv: hep-th/0603057.

[9] T. Padmanabhan, "Dark energy: mystery of the millennium", Albert Einstein Century International Conference, AIP Conf. Proc., 861, Amer. Inst. Phys., Melville, NY, 2006, 179-196; arXiv: astro-ph/0603114.

[10] M. Kaplinghat, S. Bridle, Phys. Rev. D, 71:12 (2005), 3003; arXiv: astro-ph/0312430.

[11] G. Veneziano, J. Cosmol. Astropart. Phys., 03 (2004), 004; arXiv: hep-th/0312182.

[12] S. Dubovsky, T. Gregoire, A. Nicolis, R. Rattazzi, JHEP, 03 (2006), 025; arXiv: hep-th/0512260.

[13] N. Arkani-Hamed, H. C. Cheng, M. A. Luty, S. Mukohyama, JHEP, 05 (2004), 074; arXiv: hep-th/0312099.

[14] F. Piazza, S. Tsujikawa, J. Cosmol. Astropart. Phys., 07 (2004), 004; arXiv: hep-th/0405054.

[15] A. Adams, N. Arkani-Hamed, S. Dubovsky, A. Nicolis et al., JHEP, 10 (2006), 014; arXiv: hep-th/0602178.

[16] P. Creminelli, M. A. Luty, Al. Nicolis, L. Senatore, JHEP, 12 (2006), 080; arXiv: hep-th/0606090.

[17] I. Ya. Aref'eva, "Nonlocal string tachyon as a model for cosmological dark energy", $p$-Adic Mathematical Physics, AIP Conf. Proc., 826, Amer. Inst. Phys., Melville, NY, 2006, 301-311; arXiv: astro-ph/0410443.

[18] I. Ya. Aref'eva, L. V. Joukovskaya, JHEP, 10 (2005), 087; arXiv: hep-th/0504200.

[19] G. Calcagni, JHEP, 05 (2006), 012; arXiv: hep-th/0512259.

[20] I. Ya. Aref'eva, A.S. Koshelev, JHEP, 02 (2007), 041; arXiv: hep-th/0605085; I. Ya. Aref'eva, "D-brane as a model for cosmological dark energy", Contents and Structures of the Universe, Proc. XLI Rencontres de Moriond (La Thuile, Italy, March 18-25, 2006), eds. C. Magneville, R. Ansari, J. Dumarchez, J. T. T. Van, NXB The Gioi, Hanoi, 2006, 131-135. 
[21] I. Ya. Aref'eva, P. B. Medvedev, A. P. Zubarev, Phys. Lett. B, 240:3-4 (1990), 356-362; Nucl. Phys. B, 341:2 (1990), 464-498; C. R. Preitschopf, C. B. Thorn, S. A. Yost, Nucl. Phys. B, 337:2 (1990), 363-433; I. Ya. Arefeva, D. M. Belov, A. S. Koshelev, P. B. Medvedev, Nucl. Phys. B, 638:1-2 (2002), 3-20; arXiv: hep-th/0011117.

[22] I. Ya. Aref'eva, L. V. Joukovskaya, A. S. Koshelev, JHEP, 09 (2003), 012; arXiv: hep-th/0301137; Ya.I. Volovich, J. Phys. A, 36:32 (2003), 8685-8701; arXiv: math-ph/0301028.

[23] A. Sen, Internat. J. Modern Phys. A, 20:24 (2005), 5513-5656; arXiv: hep-th/0410103.

[24] K. Ohmori, A review on tachyon condensation in open string field theories; arXiv: hep-th/0102085; I. Ya. Aref'eva, D. M. Belov, A. A. Giryavets, A. S. Koshelev et al., Noncommutative field theories and (super)string field theories, arXiv: hep-th/0111208; W. Taylor, Lectures on D-branes, tachyon condensation, and string field theory, arXiv: hep-th/0301094.

[25] В. А. Рубаков, TMФ, 149:3 (2006), 409-426; arXiv: hep-th/0604153.

[26] R. Gannouji, D. Polarski, A. Ranquet, A. A. Starobinsky, J. Cosmol. Astropart. Phys., 09 (2006), 016; arXiv: astro-ph/0606287.

[27] R. Lazkoz, R. Maartens, E. Majerotto, Phys. Rev. D, 74:8 (2006), 3510; arXiv: astro-ph/0605701.

[28] I. P. Neupane, Towards inflation and accelerating cosmologies in string-generated gravity models, arXiv: hep-th/0605265.

[29] S. Nojiri, S. D. Odintsov, M. Sami, Phys. Rev. D, 74:4 (2006), 6004; arXiv: hep-th/0605039.

[30] M. Ostrogradski, Memoires Sur Les Equations Differentielles Relatives au Probleme des Isoperimetres, VI 4, Mem. Ac. St. Petersburg, 1850.

[31] T. Nakamura, S. Hamamoto, Progr. Theoret. Phys., 95:3 (1996), 469-484; arXiv: hep-th/9511219.

[32] A. Pais, G. E. Uhlenbeck, Phys. Rev., 79:1 (1950), 145-165.

[33] И. В. Волович, Калибровочная инвариантность в аксиоматическом подходе, Дипломная работа, МГУ, 1969.

[34] В. С. Владимиров, Я.И. Волович, ТМФ, 355-368:3 (2004); arXiv: math-ph/0306018; В. С. Владимиров, Изв. РАН. Сер. матем., 69:3 (2005), 55-80; arXiv: math-ph/0507018; Л. В. Жуковская, ТМФ, 146:3 (2006), 402-409; D. V. Prokhorenko, On some nonlinear integral equation in the (super) string theory, arXiv: math-ph/0611068.

[35] I. V. Volovich, Classical Quantum Gravity, 4:4 (1987), L83-L87; И. В. Волович, ТМФ, 71:3 (1987), 337-340.

[36] L. Brekke, P. G. O. Freund, M. Olson, E. Witten, Nucl. Phys. B, 302:3 (1988), 365-402; P. H. Frampton, Ya. Okada, Phys. Rev. D, 37:10 (1988), 3077-3079.

[37] В. С. Владимиров, И. В. Волович, Е.И. Зеленов, p-Адический анализ и математическая физика, Наука, М., 1994.

[38] I. Ya. Aref'eva, L. V. Joukovskaya, S. Yu. Vernov, JHEP, 07 (2007), 087; arXiv: hep-th/0701184.

[39] В.В. Козлов, И. В. Волович, Докл. РАН, 408:3 (2006), 317-320; V. V. Kozlov, I. V. Volovich, Int. J. Geom. Methods Mod. Phys., 3:7 (2006), 1349-1357; arXiv: gr-qc/0603111.

[40] И. Я. Арефьева, А.С. Кошелев, С. Ю. Вернов, ТМФ, 148:1 (2006), 23-41; arXiv: astro-ph/0412619; I. Ya. Aref'eva, A. S. Koshelev, S. Yu. Vernov, Phys. Lett. B, 628:1-2 (2005), 1-10; arXiv: astro-ph/0505605; Phys. Rev. D, 72:6 (2005), 4017; arXiv: astro-ph/0507067.

[41] M. P. Dabrowski, C. Kiefer, B. Sandhöfer, Phys. Rev. D, 74:4 (2006), 4022; arXiv: hep-th/0605229.

[42] E. O. Kahya, V. K. Onemli, Phys. Rev. D, 76:4 (2007), 3512; arXiv: gr-qc/0612026. 